\title{
ALGUNAS APROXIMACIONES TEÓRICAS EN LA DINÁMICA DE LAS RESTRICCIONES CONSTITUCIONALES AL EJERCICIO DE LOS DERECHOS HUMANOS DENTRO DEL BLOQUE CONSTITUCIONAL EN MÉXICO
}

\author{
Some theoretical approaches in the dynamics of the constitutional human rights \\ limitations in constitutional block in Mexico
}

\section{Ángel Fernando PRADO LÓPEZ}

Sumario:

I. Introducción al bloque constitucional/convencional en México. II. Del bloque constitucional/convencional al parámetro de regularidad. Breve referencia a la contradicción de tesis 293/2011. III. Aproximaciones a la interpretación de las restricciones constitucionales dentro del bloque constitucional en México a partir de la contradicción de tesis 293/2011 . IV. Reflexiones finales. V. Fuentes de información.

Resumen: el presente trabajo analiza la dinámica de las restricciones constitucionales al ejercicio de los derechos humanos dentro del bloque constitucional en el modelo mexicano a partir de la jurisprudencia por contradicción de tesis 293/2011, sugiriendo que no toda restricción debe prevalecer sobre los derechos humanos.

Abstract: this paper analyzes the dynamics of constitutional restrictions on the exercise of human rights within the constitutional block in the Mexican model based on the case law 293/2011 contradictory argument, suggesting that not all restrictions should take precedence over human rights.

Palabras clave: bloque constitucional, derechos humanos, restricciones constitucionales, principio pro personae, cláusula de interpretación conforme.

Keywords: constitutional block, human rights, constitutional restictions, pro personae principle, under clause interpretation.

\footnotetext{
${ }^{1}$ Es Licenciando en Derecho por la Universidad de Colima y Maestro en Juicio de Amparo por la Universidad del Valle de Atemajac UNIVA Plantel Colima. Cuenta con el nombramiento de Actuario en el Tribunal de lo Contencioso Administrativo del Estado de Colima; asimismo se desempeña como docente en la UNIVA Campus Colima dentro de la Licenciatura en Derecho y de la Maestría en Juicio de Amparo con materias como: Derecho Constitucional, Derechos Humanos y Derecho Administrativo y Derecho Comparado, de igual forma ha sido profesor-asesor en la Maestría en Juicio de Amparo de la misma Institución con las materias: Derechos Humanos y Garantías Constitucionales y Metodología de la Jurisprudencia y la Legislación; es Profesor Adjunto en la Facultad de Derecho de la Universidad de Colima con las materias: Derecho Administrativo y Derechos Humanos; ha publicado diversos artículos en revistas especializadas y realizado diversos cursos y diplomados con temática de Derechos Humanos, recientemente concluyó exitosamente el curso “International Human Rights” impartido por la Universidad Católica de Lovaina de Bélgica.
} 


\section{INTRODUCCIÓN AL BLOQUE CONSTITUCIONAL/CONVENCIONAL EN MÉXICO}

Los últimos cuatro años han supuesto una serie de cambios importantes y exponenciales en el sistema jurídico mexicano a partir de dos momentos cruciales: por un lado el ya muy conocido caso Rosendo Radilla vs. México, cuya sentencia derivó en la formación del expediente varios 912/10 en la Suprema Corte de Justicia de la Nación teniendo grandes implicaciones en la impartición de la justicia en México, entre otros con la implementación del control difuso de convencionalidad por las autoridades del Poder Judicial; y en otro tenor, la reforma constitucional en materia de derechos humanos ${ }^{2}$ del año de 2011; ambos son los orígenes de un nuevo cauce en la interpretación del derecho en el contexto mexicano.

La obligación por ejemplo, de ejercer el control difuso de convencionalidad por todas las autoridades que imparten justicia; robustecer el catálogo de derechos humanos provenientes de fuentes nacionales y extranjeras, así como marcar la ruta de la interpretación de aquellos derechos a través de la cláusula de interpretación conforme y el principio pro persona (ahora ya constitucionalizados), enmarcan un escenario que desemboca en un paradigma novedoso y de necesario análisis que permita llevar a la práctica la reforma mencionada.

Este trabajo se referirá particularmente a la integración en el sistema jurídico mexicano lo que en la doctrina del derecho comparado se conoce como bloque de constitucionalidad o para seguir al Dr. Diego Valadés bloque constitucional, ${ }^{3}$ término adoptado por la Suprema Corte de Justicia mexicana como parámetro de control de regularidad constitucional. ${ }^{4}$

Hablar del bloque constitucional remite directamente a su origen, siendo éste un concepto de procedencia francesa que hace referencia a un reenvío normativo desde la Constitución como documento jurídico-político supremo de un país a otras normas de diversa fuente, y que como consecuencia de esa remisión adquieren una misma jerarquía a la de la norma fundamental, es decir, se trata de un concepto en donde se amplía el contenido constitucional pues el enfoque es material más que formal, expandiéndose los alcances de la Carta Magna a otros órdenes que no tienen su nacimiento propiamente en la máxima norma, pero que adquieren ese rango (el de norma suprema en cuanto parámetro de validez) en virtud de lo expresado por ella misma.

$\mathrm{Al}$ respecto la Mtra. Graciela Rodríguez Manzo 5 señala lo siguiente:

...en términos generales podemos sostener que se trata de una categoría jurídica (un concepto) del derecho constitucional comparado que se refiere al conjunto de normas que tienen jerarquía constitucional en el ordenamiento jurídico de cada país; así, el bloque de constitucionalidad parte del supuesto según el cual "las normas constitucionales no son sólo aquellas que aparecen expresamente en la Carta sino también aquellos principios y

\footnotetext{
${ }^{2}$ En el presente trabajo se tienen en cuenta las diferencias y precisiones entre los conceptos derechos humanos y derechos fundamentales; sin embargo para efectos prácticos se utilizarán como sinónimos en este artículo.

${ }^{3}$ El Dr. Valadés precisó que se trata de un bloque constitucional más que de constitucionalidad al distinguir a éste último como aquello conforme solo con la Constitución propiamente dicha, esto dentro del marco del IX Encuentro Iberoamericano y VI Congreso Mexicano de Derecho Procesal Constitucional celebrado en el mes de diciembre de 2014 en la ciudad de Hermosillo Sonora, México.

${ }^{4}$ Más adelante se desarrollarán algunas de sus consecuencias.

5 Bloque de Constitucionalidad en México, http://www2.scjn.gob.mx/red/coordinacion/Bloque\%2ode\%20 constitucionalidad.pdf, 19 de enero de 2015.
} 
valores que no figuran directamente en el texto constitucional pero a los cuales la propia Constitución remite.

En ese orden de ideas y siguiendo a la misma autora, una vez conformado el bloque a través de ese reenvío normativo se convierte en el parámetro de interpretación de todo el ordenamiento jurídico, a la vez que se vuelve el parámetro de validez de todo acto de autoridad, destacando la nula importancia de la fuente utilizada para dicha interpretación, es decir, las fuentes de referencia en estos ejercicios crecen, se amplían e incluyen tanto a las de sede interna como las de sede externa.

En México con el texto constitucional ya reformado en el año de 2011, cabe cuestionarse desde un inicio si se considera la existencia de un bloque constitucional reconocido por la norma fundamental y para responder es necesario revisar algunos de sus contenidos comenzando por los dos primeros párrafos del artículo $1^{\circ}$ :

Artículo 1o. En los Estados Unidos Mexicanos todas las personas gozarán de los derechos humanos reconocidos en esta Constitución y en los tratados internacionales de los que el Estado Mexicano sea parte, así como de las garantías para su protección, cuyo ejercicio no podrá restringirse ni suspenderse, salvo en los casos y bajo las condiciones que esta Constitución establece.

Las normas relativas a los derechos humanos se interpretarán de conformidad con esta Constitución y con los tratados internacionales de la materia favoreciendo en todo tiempo a las personas la protección más amplia.

El primero de los anteriores párrafos habla del reconocimiento de los derechos humanos tanto en la norma fundamental como los establecidos en los tratados internacionales de los que el Estado Mexicano es parte, evidenciando de inicio un reenvío normativo que parte de la misma Constitución hacia los tratados internacionales, traduciéndose en un robustecimiento del catálogo de derechos humanos que se extiende más allá de los límites del texto constitucional, formando de esta manera ese bloque o ese conjunto normativo de derechos humanos que se visualiza como un todo y no de manera aislada, implicando lo siguiente:

- Se integran a la Constitución los derechos humanos originados en otras fuentes además de las nacionales;

- No sólo amplía este catálogo de derechos humanos, sino que a la vez reune las garantías necesarias para su resguardo y protección;

- De la lectura literal se configura una lógica de contenido o sustancia del derecho humano por su objeto y fin mas no así de la jerarquía de la fuente que lo genera, en consecuencia se desvanece cualquier tipo de grado entre las fuentes de los derechos humanos, y finalmente;

- El bloque se conforma como un conjunto de derechos y garantías vistos de manera holística, dejando de lado la clásica idea muy arraigada en la doctrina mexicana de tener el apartado dogmático constitucional limitado al articulado de ese texto solamente, y como 
consecuencia se puede hablar de un bloque constitucional / convencional formado por la Constitución - tratados internacionales.

Respecto al segundo párrafo del citado artículo es importante señalar una idea que comenzó a desarrollar el Dr. José Luis Caballero Ochoa, y que está relacionada con la aproximación de un segundo bloque de interpretación resultado de este párrafo. En líneas anteriores se comentó cómo el bloque constitucional/convencional se forma por el reenvío normativo expreso en la Carta Fundamental, sin embargo, ese es un primer reenvío integrado por las distintas fuentes de los derechos humanos que funciona como parámetro de validez del ordenamiento jurídico (y dada la transversalidad de los derechos fundamentales a todos los campos del derecho) complementándose aquél con un segundo bloque en el párrafo subsecuente, este último conformado por la interpretación del primer bloque, aquí de nuevo se expone: las normas relativas a los derechos humanos se interpretarán de conformidad con esta Constitución y con los tratados internacionales de la materia favoreciendo en todo tiempo a las personas la protección más amplia. Siguiendo la idea mencionada se observa la existencia de un bloque como consecuencia de la interpretación de los derechos humanos que manejan su ruta a través de la cláusula de interpretación conforme y del principio pro persona, los cuales sirven para dar contenido a aquéllos que derivan del binomio constitucióntratados internacionales, ${ }^{6}$ materialmente los derechos humanos toman forma por conducto de las resoluciones o bien de la jurisprudencia cuyo papel es definirlos a partir del piso de protección estándar o básico en la norma de derecho doméstico como la de origen externo para mejorarlos progresivamente.

$\mathrm{Al}$ establecer la norma constitucional que los derechos humanos se interpretan a través de sus propios contenidos y de los tratados internacionales por medio del principio pro persona y de la interpretación conforme, se marcan las rutas de la manera en cómo se dotará de materia al o los derechos humanos en el caso concreto, volviéndose parámetro de interpretación del ordenamiento jurídico. Esto desde luego maximiza o potencia el contenido esencial del derecho bajo claves interpretativas derivadas del reenvío normativo del propio bloque constitucional/convencional, por ello se hace referencia a la posibilidad de tener dos bloques, uno previo como resultado del modelo integrativo señalado en la Constitución, y otro posterior derivado de la interpretación de los derechos humanos a través de los desarrollos jurisprudenciales en el esquema del binomio constitución-tratados internacionales.

Cabe mencionar que la utilización del ejercicio hermenéutico de la interpretación conforme a través del binomio referido, de inicio permite incorporar al sistema jurídico mexicano las distintas fuentes del derecho internacional de los derechos humanos, de ahí que las claves de interpretación dejan de ser exclusivamente aquellas referidas a la Constitución, y a la vez impacta y desarrolla los alcances de las obligaciones a cargo de las autoridades en materia de derechos humanos, así como de los principios alrededor de ellos. A ello se refiere el tercer párrafo del artículo $1^{\circ}$ constitucional que a la letra señala:

Todas las autoridades, en el ámbito de sus competencias, tienen la obligación de promover, respetar, proteger y garantizar los derechos humanos de conformidad con los principios de universalidad, interdependencia, indivisibilidad y progresividad. En consecuen-

\footnotetext{
${ }^{6}$ CABALLERO OCHOA, José Luis, "La interpretación conforme, el modelo constitucional ante los tratados internacionales sobre derechos humanos y el control de convencionalidad” $2^{\text {a }}$ ed., Porrúa, México, 2014, pp. 251-252.
} 
cia, el Estado deberá prevenir, investigar, sancionar y reparar las violaciones a los derechos humanos, en los términos que establezca la ley.

En tal virtud el bloque constitucional/convencional no puede dejar fuera los contenidos de los que se deben dotar también a las obligaciones (promover, respetar, proteger y garantizar) ni los principios (universalidad, interdependencia, indivisibilidad y progresividad) de los derechos humanos para formar ese sistema integrativo que parte desde la Constitución siguiendo la línea de un modelo de Estado Democrático Constitucional y que de alguna manera la existencia de este conjunto de derechos permite un mejor acercamiento del texto fundamental a la realidad social, al dinamizar estas normas provenientes de diversas fuentes para proveer un desarrollo más adecuado a los derechos humanos; en todo caso lo interesante es saber cómo fue adoptada esta doctrina del bloque en la interpretación de nuestro máximo tribunal, y a ello se dedican las próxima líneas. ${ }^{7}$

\section{DEL BLOQUE CONSTITUCIONAL/CONVENCIONAL AL PARÁMETRO DE REGULARIDAD. BREVE REFERENCIA A LA CONTRADICCIÓN DE TESIS 293/11}

En el apartado anterior se trató de esclarecer el concepto del bloque constitucional/convencional, haciendo énfasis al modelo mexicano teniendo como punto de partida el texto constitucional mediante el cual se hace una remisión expresa a los tratados internacionales en materia de derechos humanos (siempre y cuando México sea parte de esos tratados) y su consecuente interpretación, sirviendo aquél como parámetro de validez de todo acto de autoridad.

Una de las implicaciones de acogernos a ese concepto es la de eliminar cualquier tipo de jerarquía entre las diversas fuentes que conforman el bloque; es decir, al establecer las rutas de interpretación a través del principio pro persona y la cláusula de interpretación conforme, la idea de grados o niveles entre los derechos contenidos en la norma nacional y los de las normas internacionales se desvanecen, en función de encontrar la protección más amplia del derecho humano en juego (principio pro persona), así el bloque se materializa en el momento que los derechos humanos se conciben en claves de su contenido o sustancia, desde ejercicios interpretativos axiológicos y no de jerarquía, y así es como de la lectura literal de los párrafos primero y segundo del artículo $1^{\circ}$ constitucional se explica el bloque.

Sin embargo ha sido característica de la doctrina jurídica en México interpretar los derechos a la luz de un esquema vertical y piramidal, generando como consecuencia que el contexto de un bloque de derechos donde se eliminan las jerarquías de sus fuentes sufra de enorme reticencia por gran parte de los operadores jurídicos y otro tanto por un sector de la academia; parecería que importa más si el derecho humano se concibe y protege desde la Constitución y no así aquel originado en sede externa, abrazando el concepto de supremacía constitucional a límites en donde ya no es posible aplicarlo en virtud de la integración del derecho internacional de los derechos humanos al ordenamiento jurídico mexicano. ${ }^{8}$

Lo anterior trajo como consecuencia la generación de dos posturas disímiles en el entendimiento de lo que el artículo $1^{\circ}$ constitucional vinculado al 133 de la misma norma su-

\footnotetext{
7 No se debe perder de vista que el bloque constitucional también se encuentra previsto por otros artículos dentro de la Constitución Mexicana, tales como los numerales: 15, 103, 105 y 107.

$8 \mathrm{Me}$ parece necesario transcribir nuevas aproximaciones sobre el concepto de supremacía constitucional, citada por el Dr. José Luis Caballero Ochoa, entendiéndola: no solo como el ordenamiento que determina las
} 
gerían como una nueva lectura del concepto de supremacía tratándose de las fuentes de los derechos humanos. De esta manera y sin hacer referencia al desarrollo de cada uno de estos extremos, se forma el expediente de contradicción de tesis 293/2011 resuelto por la Suprema Corte de Justicia de la Nación en el último semestre del año de 2013 y cuyo engrose fue publicado seis meses después, el cual refleja algunos puntos importantes de la discusión que se pueden resumir en estas interrogantes: ¿Qué lugar ocupan dentro de la jerarquía de normas en el sistema jurídico mexicano, los tratados internacionales? ¿Las normas de derechos humanos se interpretan en términos jerárquicos?; ¿Existe un bloque constitucional/ convencional derivado de la Constitución Mexicana?

Estos cuestionamientos preocuparon a los Ministros de la Suprema Corte al tratar de marcar una ruta que extrajera del limbo jurídico principalmente a los operadores jurídicos sobre cómo se debía entender lo señalado por el artículo $1^{\circ}$ constitucional; llegar al consenso no fue sencillo y de hecho produjo uno de los criterios más cuestionados y polémicos dentro del activismo de la Corte, quedando como sigue:

DERECHOS HUMANOS CONTENIDOS EN LA CONSTITUCIÓN Y EN LOS TRATADOS INTERNACIONALES. CONSTITUYEN EL PARÁMETRO DE CONTROL DE REGULARIDAD CONSTITUCIONAL, PERO CUANDO EN LA CONSTITUCIÓN HAYA UNA RESTRICCIÓN EXPRESA AL EJERCICIO DE AQUÉLLOS, SE DEBE ESTAR A LO QUE ESTABLECE EL TEXTO CONSTITUCIONAL. El primer párrafo del artículo 1o. constitucional reconoce un conjunto de derechos humanos cuyas fuentes son la Constitución y los tratados internacionales de los cuales el Estado Mexicano sea parte. De la interpretación literal, sistemática y originalista del contenido de las reformas constitucionales de seis y diez de junio de dos mil once, se desprende que las normas de derechos humanos, independientemente de su fuente, no se relacionan en términos jerárquicos, entendiendo que, derivado de la parte final del primer párrafo del citado artículo 1o., cuando en la Constitución haya una restricción expresa al ejercicio de los derechos humanos, se deberá estar a lo que indica la norma constitucional, ya que el principio que le brinda supremacía comporta el encumbramiento de la Constitución como norma fundamental del orden jurídico mexicano, lo que a su vez implica que el resto de las normas jurídicas deben ser acordes con la misma, tanto en un sentido formal como material, circunstancia que no ha cambiado; lo que sí ha evolucionado a raíz de las reformas constitucionales en comento es la configuración del conjunto de normas jurídicas respecto de las cuales puede predicarse dicha supremacía en el orden jurídico mexicano. Esta transformación se explica por la ampliación del catálogo de derechos humanos previsto dentro de la Constitución Política de los Estados Unidos Mexicanos, el cual evidentemente puede calificarse como parte del conjunto normativo que goza de esta supremacía constitucional. En este sentido, los derechos humanos, en su conjunto,

formas de producción normativa, sino la aplicación de otros ordenamientos no producidos desde el ordenamiento interno. Un centro de convergencia en unidad, más que un vértice del cual todo deriva y marca el punto de partida de la ley, al tener en consideración los contenidos normativos que convergen en el orden jurídico nacional. De esta forma, en el desarrollo jurídico comparado, el principio de supremacía constitucional es un principio que permite ordenar las competencias; la producción normativa, o la aplicación de órdenes normativos complejos y plurales; en CABALLERO OCHOA, José Luis, Op. Cit., nota 3, p. 134. 
constituyen el parámetro de control de regularidad constitucional, conforme al cual debe analizarse la validez de las normas y actos que forman parte del orden jurídico mexicano. ${ }^{9}$

De la citada jurisprudencia se realizan los siguientes planteamientos: ¿Qué entendió la Corte cuando señala que las normas de derechos humanos independientemente de su fuente no se relacionan en términos jerárquicos, si posteriormente se refiere a la supremacía constitucional en el tema de las restricciones al ejercicio de los derechos humanos? ¿Tiene implicaciones prácticas hablar de parámetro de regularidad constitucional y no de bloque constitucional? ¿Existe contradicción en el contenido de la jurisprudencia en sí misma? y finalmente ¿Qué son las restricciones expresas al ejercicio de los derechos humanos?

Se responderá en el mismo orden formulado con las siguientes acotaciones:

a) Parece haber un primer avance en la jurisprudencia transcrita, pues al inicio de la misma se deja en claro que las normas referentes a los derechos humanos no deben interpretarse en claves de jerarquía, pues carece de importancia la fuente de la cual provengan sea nacional o internacional, con lo cual se confirma como guía de interpretación el principio pro persona y la cláusula de interpretación conforme, dejando atrás la arraigada idea de la percepción de los derechos humanos desde un enfoque vertical y se recoge bajo una ruta axiológica de los propios derechos; el problema sin embargo, podría radicar en lo que más adelante señala este criterio respecto de las restricciones expresas al ejercicio de los derechos humanos donde prevalecerá la restricción constitucional y no el derecho en sí mismo. Se sugiere entonces que el concepto de jerarquía o de escala no desaparece del todo, ya que en el tema de las restricciones se inserta una aproximación en donde se sigue utilizando un acercamiento piramidal bastante obsoleto, en virtud de lo mencionado en el primer apartado de este artículo al haberse integrado al bloque constitucional los derechos humanos contenidos en los tratados internacionales, añadiéndose también los valores, principios y reglas de interpretación propias del derecho internacional de los derechos humanos, estas viejas fórmulas de grado que ocupa una norma sobre otra terminan sobrando.

b) Por lo que atañe al parámetro de control de regularidad constitucional, parecería no existir algún tipo de impacto para efectos de su aplicación práctica, sin embargo César Astudillo citado por José Luis Caballero Ochoa, ${ }^{10}$ menciona cómo la idea de bloque sí difiere de la de parámetro, pues en la primera se entiende un conjunto de derechos desde una óptica sustantiva de contenido de los mismos, mientras el parámetro se acota solamente a reglas procesales; aún con estas precisiones lo cierto es que para la Corte la idea de parámetro funciona como referente bajo el cual se deben interpretar las normas de los derechos humanos, además de funcionar como estándar de validez de todo acto de autoridad, ya que tendrán que estar de acuerdo con el binomio Constitución-tratados internacionales.

c) Desde este trabajo se afirma la existencia de una contradicción en el contenido de la jurisprudencia resultado del expediente 293/2011, pues en un primer momento se señala

9 Época: Tesis: P./J. 20/2014 (10a.), Gaceta del Semanario Judicial de la Federación, Décima Época Libro 5, Abril de 2014, Tomo I Materia(s): Constitucional p. 202.

${ }^{10}$ CABAllero OCHOA, José Luis, Op. Cit., nota 3, p. 250. 
cómo las normas de derechos humanos no se relacionan en términos de jerarquía independientemente de su fuente, aunque posteriormente hace prevalecer a las restricciones expresas en la Constitución al ejercicio de los derechos humanos. La palabra prevalecer por sí misma refiere a dar un valor de mayor importancia al texto constitucional que al contenido de un tratado internacional, se vuelve una regla en apariencia a priori de la manera cómo se entenderán y resolverán las restricciones dentro de la Norma Fundamental, aún cuando la misma jurisprudencia reconoce al mismo tiempo la conformación del parámetro de regularidad, por lo tanto es pertinente preguntarse también si en el reenvío normativo hacia otros órdenes utilizando la interpretación conforme y el principio pro persona quedarán desplazados o derrotados los derechos humanos ante una restricción expresa dentro de la Carta Máxima.

Lo anterior finalmente permite desarrollar el tema de las restricciones en esa interacción dentro del bloque constitucional/convencional, materia principal de reflexión del presente trabajo y que se desenvuelve en los siguientes apartados.

III. APROXIMACIONES A LA INTERPRETACIÓN DE LAS RESTRICCIONES CONSTITUCIONALES DENTRO DEL BLOQUE CONSTITUCIONAL EN MÉXICO A PARTIR DE LA CONTRADICCIÓN DE TESIS 293/2011

Algunas de las disertaciones señaladas anteriormente servirán para apuntalar la postura particular que se tiene respecto al tema de las restricciones al ejercicio de los derechos humanos. Se hizo hincapié en cómo la jurisprudencia nacida de la contradicción de tesis 293/2011 parece caer en una serie de juegos de palabras donde lo primero termina por contradecir a lo último, por lo que se dan pasos agigantados para confirmar la existencia de un bloque de derechos que elimina la jerarquía de sus fuentes, pero en el tema de las restricciones al ejercicio de los derechos fundamentales, tajantemente sí se les interpreta desde la óptica vertical y piramidal (jerarquía) sumamente enraizada en un amplio sector de la comunidad jurídica en México.

No se trata de un tema sencillo ni mucho menos, pues los límites de los derechos humanos se configuran para poder mantener un cierto orden en el ejercicio de los mismos, esto es, para identificar dentro de cuáles estándares los derechos fundamentales pueden dinamizarse para su disfrute por cada una de las personas, sin excederse en su ejecución al grado de afectar los derechos de otros sujetos, derivando ello también en una práctica del poder público para mantener ese orden dentro de una sociedad cualquiera, originándose aquí una coyuntura importante: ningún derecho humano es absoluto.

Sin embargo habrá que acotar algunas ideas, la primera de ellas se refiere a la distinción entre delimitar y limitar/restringir un derecho, ${ }^{11}$ y para esclarecerlo se cita a Claudia Alejandra Villaseñor Goyzueta. La referida autora diferencia ambos conceptos y entiende a la delimitación como parte de la definición del derecho humano en sí mismo, es decir, se trata de un marco interno inherente a la manera en cómo se establecen ciertos cauces en el bien o valor protegido por el derecho; y en tanto los límites como condicionamientos externos al

\footnotetext{
${ }^{11}$ Aquí hago la aclaración que la expresión restricciones se utilizará en el sentido de los límites propiamente. 
derecho enunciado en abstracto ${ }^{12}$ con el objetivo de posibilitar su goce por los miembros del colectivo social, sin desdibujar su naturaleza.

De esta manera, las restricciones/límites a los derechos fundamentales: son normas que restringen su objeto de protección, y por ello tan solo podrán ser adoptados de conformidad con la Constitución. Estos límites suponen la adopción de normas que establecen obligaciones $y$ prohibiciones en relación al objeto de protección del derecho fundamental. ${ }^{13}$

La definición anterior permite objetar el contenido de la jurisprudencia emitida por la Suprema Corte de México en la contradicción de tesis 293/2011, en concreto el apartado donde se señala la prevalencia de las restricciones en la Carta Fundamental y no así de los derechos humanos; resulta un tema complejo y que genera las siguientes interrogantes: ¿Cuál es el papel del bloque constitucional/convencional en la interpretación de las restricciones al ejercicio de los derechos humanos?; ¿En virtud de ese bloque cómo operan las reglas de la interpretación conforme y el principio pro persona respecto de las restricciones constitucionales?; ¿Cuál podría ser el vínculo del principio de progresividad de los derechos humanos para derrotar o no algunas restricciones constitucionales?; y finalmente ¿Tendrían que aceptarse como constitucionales cualquier tipo de restricciones solo por razón de estar plasmadas en el texto fundamental, o podría hablarse de una restricción por sí misma inconstitucional?

A continuación se desglosan las respuestas en los siguientes párrafos:

A) El papel del bloque / parámetro de control de la constitucionalidad resulta de especial interés para entender la dinámica de las restricciones dentro de él. Como se comentó al inicio del presente trabajo el bloque de derechos se conforma a través del reenvío normativo del texto constitucional hacia otros órdenes originados fuera de éste pero que adquieren rango fundamental por disposición expresa de la Constitución. La formación de ese catálogo de derechos servirá de piso mínimo para el respeto, la promoción, la protección y la garantía de los derechos humanos, así como de los instrumentos para su resguardo, extendiendo también esta interpretación a las obligaciones a cargo de las autoridades públicas para potenciar el contenido de los derechos humanos en aras de un mejor disfrute de los mismos.

Ahora bien, como sistema autointegrativo de derechos (en palabras de Argelia Queralt) ¿Qué sucede cuando el poder reformador de la Constitución introduce una restricción al ejercicio de un derecho humano totalmente desproporcionada? ${ }^{14}$ en virtud del bloque podría suponerse que uno de los poderes (en este caso el legislativo-constituyente permanente) en el ámbito de su competencia incumple con el párrafo tercero del artículo $1^{\circ}$ constitucional en el sentido de no respetar o garantizar debidamente un derecho humano, al excederse en la conformación y definición de la restricción, porque es justamente en la remisión de normas hacia otros órdenes y a su interpretación que no solo dota de contenido al derecho humano en cuestión, sino a la vez determina los alcances de las obligaciones referidas antes, con lo cual se deduce observándolo desde esta arista, una violación a la Constitución por partida

\footnotetext{
${ }^{12}$ VILLASEÑOR GOYZUETA, Claudia Alejandra, "Proporcionalidad y límites de los derechos fundamentales, teoría general y su reflejo en la jurisprudencia mexicana”, $1^{\mathrm{a}}$ ed., Porrúa, Escuela Libre de Derecho y Universidad Complutense, México, 2011, p. 44.

${ }^{13}$ Idem.

${ }^{14}$ En apartados siguientes se explicará el principio de proporcionalidad.
} 
doble: por un lado al incumplir con su mandato en cuanto a las obligaciones a cargo de las autoridades; y por otro en términos de la restricción en sí misma que altera el principio de unidad de la Constitución. ${ }^{15}$ Sin embargo nuestro Máximo Tribunal parece seguir fielmente la línea del parámetro de regularidad, tal cual se estableció en la contradicción de tesis $293 / 2011 .^{16}$

B) Oportuno es precisar ahora de qué forma la interpretación conforme y el principio pro persona interactúan dentro del bloque constitucional/convencional en el tema de las restricciones de los derechos, particularmente como lo señala José Luis Caballero Ochoa el modelo mexicano de interpretación de conformidad con la Constitución, genera las siguientes implicaciones para dicho ejercicio:

I. De todas las normas relativas a los derechos humanos contenidas en la legislación, hacia los referentes Constitución y los tratados internacionales.

II. De las normas sobre derechos humanos contenidas en los tratados internacionales hacia la Constitución y los tratados internacionales.

III. De las normas sobre derechos humanos contenidas en la Constitución, hacia otras normas de la Constitución y los tratados internacionales. ${ }^{17}$

Como se observa, la interpretación conforme como ejercicio hermenéutico para integrar y armonizar los derechos humanos de sede interna al derecho internacional de los derechos humanos (con sus valores, principios y reglas de interpretación), confirma la configuración de un bloque de derechos desde el modelo mexicano creado por la propia Constitución. Es de especial interés destacar la tercera de las consecuencias de interpretar conforme a la Constitución, primeramente porque hacerlo lleva consigo entender que por Constitución se deduce ésta junto con los tratados internacionales, no solo al documento de 136 artículos, pues se trata de un binomio inescindible en palabras del Dr. José Luis Caballero Ochoa, y visualizándolo de esa manera (Constitución-tratados internacionales) las normas de derechos humanos operan dentro del bloque hacia la propia Constitución, esto significa que el contenido de la norma fundamental con respecto al resto de sus normas deben estar acordes

\footnotetext{
${ }^{15}$ Este ejemplo podría tener sus detractores al señalar que el poder reformador está por encima de los demás poderes, cuestión que desde mi perspectiva es incorrecta, porque no puede ser superior al contenido constitucional y no deja de ser un poder constituido.

${ }^{16}$ DERECHOS HUMANOS RECONOCIDOS TANTO POR LA CONSTITUCIÓN POLÍTICA DE LOS ESTADOS UNIDOS MEXICANOS, COMO EN LOS TRATADOS INTERNACIONALES. PARA DETERMINAR SU CONTENIDO Y ALCANCE DEBE ACUDIRSE A AMBAS FUENTES, FAVORECIENDO A LAS PERSONAS LA PROTECCIÓN MÁS AMPLIA. Acorde con lo sostenido por el Tribunal en Pleno de la Suprema Corte de Justicia de la Nación, en la tesis de jurisprudencia P./J. 20/2014 (10a.) (*), las normas de derechos humanos contenidas en los tratados internacionales y en la Constitución Política de los Estados Unidos Mexicanos no se relacionan entre sí en términos jerárquicos, ya que se integran al catálogo de derechos que funciona como un parámetro de regularidad constitucional. Por tanto, cuando un derecho humano esté reconocido tanto en la Constitución Federal, como en los tratados internacionales, debe acudirse a ambas fuentes para determinar su contenido y alcance, favoreciendo en todo tiempo a las personas la protección más amplia; en el entendido de que cuando exista en la Constitución una restricción expresa al ejercicio de un derecho humano, se deberá estar a lo que indica la norma constitucional.

${ }^{17}$ CABALlERO OCHOA, José Luis, Op. Cit., nota 3, p. 252.
} 
al binomio de derechos señalado, impactando en consecuencia el tema de las restricciones al ejercicio de los derechos humanos. ${ }^{18}$

En este orden de ideas no se debe dejar fuera que las rutas de interpretación de los derechos se hacen también a través del principio pro persona constitucionalizado en el segundo párrafo del artículo primero de la Carta. Dicha herramienta que interactúa y complementa a la cláusula de interpretación conforme, permite aplicar la norma más favorecedora al caso concreto o aquella que proteja mejor el derecho humano en cuestión, independientemente de su fuente; sin embargo esa es solo la primera vertiente del comentado principio, en una segunda aterrizada a los límites de los derechos se deberá optar por la norma con mayores requisitos para su restricción, esto en sintonía al bien o valor jurídicamente protegido, pues a diferencia de la interpretación amplia de un derecho, tratándose de límites a los derechos la interpretación, se hace de forma restringida para afectar lo menos posible el goce del derecho humano. Lo anterior no resulta menor porque permitiría al operador jurídico muy posiblemente derrotar una restricción y no el derecho humano, pese a lo señalado por la jurisprudencia de la Corte, porque aún con esta desafortunada manera de entender al texto fundamental desde una óptica formalista y vertical, es posible reconducir a través del principio pro persona este enfoque restrictivo hacia los límites y hacer prevalecer al derecho humano. Evidentemente tendrá que ajustarse a la interpretación del caso concreto bajo esta perspectiva, sin embargo a través del control de convencionalidad cabría esta posibilidad de inaplicar en el modelo difuso la restricción, independientemente de encontrarse en la norma fundamental.

Se agrega por otro lado que las restricciones se concretan al ejercicio de los derechos humanos, es decir, el derecho humano en sí mismo no puede suspenderse como tal, solo estrictamente su ejercicio, y en este sentido el principio pro persona establecerá el alcance o los límites en la ejecución de la restricción, incluso existen derechos humanos que bajo ninguna circunstancia pueden restringirse o suspenderse en su ejercicio (tal como lo señala el artículo 29 constitucional), por lo tanto la modulación interpretativa atenderá a qué tipo de límite se trata, cuál es su justificación, su legitimidad, su objeto y si es idóneo o no.

C) Relacionado con lo anterior, los derechos humanos se desenvuelven a través de otros principios para dotarles de contenido y alcance, algunos de ellos se encuentran en el tercer párrafo del artículo $1^{\circ}$ constitucional siendo éstos: el principio de universalidad, de interdependencia, de indivisibilidad y de progresividad. Concretamente se destaca el último de ellos con dos de sus características: la gradualidad y el progreso. Sandra Serrano y Daniel Vázquez señalan que la gradualidad se refiere a que la efectividad de los derechos no se logrará de una vez y para siempre, sino que se trata de un proceso que supone definir metas a corto, mediano y largo plazos. El progreso evidencia que el disfrute de los derechos siempre

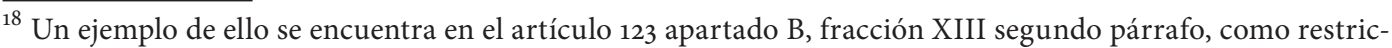
ción expresa a la posibilidad de ser los agentes del Ministerio Público, los peritos y los miembros de las instituciones policiales de la Federación, el Distrito Federal, los Estados y los Municipios, reinstalados en caso de que los Tribunales Administrativos determinen que su separación fue injustificada; aquí evidentemente no se está delimitando un derecho humano al trabajo y a los derechos sociales de los trabajadores, sino que se trata de una restricción expresa la cual no se encuentra de conformidad con la Constitución-tratados internacionales (me refiero como bloque constitucional ya que el artículo $1^{\circ}$ de la Carta Magna determina el estándar mínimo de protección de los derechos fundamentales), es desproporcionada y afecta el contenido esencial del derecho; sin embargo en la línea de la jurisprudencia de la Corte, se tendría que hacer prevalecer la restricción al ejercicio del o los derechos humanos en el caso concreto.
} 
debe mejorar ${ }^{19}$ El principio de progresividad parte de un piso de mínimo de protección del derecho humana para paulatinamente ir mejorando su disfrute, y ese estándar básico se encuentra en el artículo primero constitucional, ya más de una vez comentado. También es oportuno tener presente que la progresividad va de la mano con otro principio, el de prohibición de regresión, el cual se traduce en no dar pasos hacia atrás en detrimento de un derecho previamente reconocido, es decir, si ya se ha logrado garantizar el disfrute de un derecho humano, el Estado tiene la obligación de mejorarlo, más no así de disminuirlo.

Existe también otra interrogante relacionada con el sentido de la progresividad de los derechos: ¿qué debe prevalecer cuando un derecho humano ya se encuentra reconocido y dotado de contenido dentro de un estándar mínimo de protección, y posteriormente se introduce una restricción al ejercicio de ese derecho? Esto es, bajo un factor de temporalidad qué debe prevalecer y qué debe superarse, y se insiste, muy a pesar de la interpretación de la Suprema Corte.

Aquí vale la pena señalar que las restricciones o límites a los derechos, además de estar de conformidad con la Constitución deben cumplir por lo menos con otros dos requisitos: el principio de proporcionalidad y respetar el contenido esencial del derecho humano. El primero de ellos como lo señala Claudia Alejandra Villaseñor, se refiere a que en materia de derechos fundamentales, la existencia de un bien constitucionalmente protegido que colisiona con un derecho fundamental no justifica que se armonice la situación con total discrecionalidad. Debe evitarse que el derecho sea sacrificado injustamente. ${ }^{20}$ La proporcionalidad implica analizar qué tan apta o no es la restricción a introducir para la obtención del fin perseguido, es decir, si se trata de la medida idónea para ello, todo esto para ponderar y hacer un balance entre costos del bien sacrificado y los beneficios respectivos; ante tales ejercicios la restricción al ejercicio del derecho humano deberá interpretarse en el caso concreto y no simplemente a priori prevalecerla.

En relación al contenido esencial del derecho, además de atender al principio de proporcionalidad, se debe analizar también si en el derecho humano que se pretende restringir, se respeta ese mínimo de contenido sustancial sin el cual ese derecho quedaría desnaturalizado en su esencia, definiéndose este concepto en del derecho comparado de la siguiente manera:

Por "contenido esencial" cabría entender el contenido de los derechos constitucionalmente declarado, que debe ser delimitado por el intérprete a la luz de los preceptos constitucionales, a través de una interpretación sistemática y unitaria de la Constitución, y mediante una comprensión de cada derecho fundamental en conexión con las finalidades a las que obedece su protección.

\footnotetext{
19 SERRANO, Sandra y VAZQUEZ, Daniel, Los derechos humanos en acción, obligaciones y principios de derechos humanos. $1^{\mathrm{a}}$. ed. FLACSO, México, 2013. p. 109.

${ }^{20}$ VILLASEÑOR GOYZUETA, Claudia Alejandra, Op. Cit., nota 8, p. 59-6o. 
...eso que la Constitución denomina contenido esencial, parece evidente que no es otra cosa que el reducto último que compone la sustancia del derecho, disuelto el cual...el derecho deja de ser aquello a lo que la norma fundamental se refiere. ${ }^{21}$

Tanto el principio de proporcionalidad, como el contenido esencial funcionan como garantías para no trasgredir la esencia o sustancia del derecho humano al cual se pretende restringir, precisamente en atención a seguir manteniendo su disfrute y mejorarlo a través del principio de progresividad; es por esta razón que no se debe establecer una regla a priori en materia de restricciones constitucionales.

D) Finalmente queda una de las batallas tal vez más arduas en los derroteros de los tribunales y la academia, concretamente se relaciona con el significado de lo que se considera constitucional, es decir, por mucho tiempo en la teoría de la constitución se han construido conceptos donde se define al texto fundamental como una norma suprema que organiza al estado; posteriormente en el constitucionalismo del siglo XX resultó esencial entender a las constituciones necesariamente con un contenido robusto de derechos y sus respectivas garantías de protección, muchas de ellas bajo un esquema o modelo democrático.

Hablar del contenido de la constitución no es asunto menor, y conduce a una de las tradiciones más arraigadas del propio constitucionalismo y es la de considerar como constitucional aquello que está en la norma suprema solo por el hecho de constar en ella.

Sin embargo con el paso del tiempo la idea de lo que debe ser considerado constitucional ha evolucionado a un aspecto más sustantivo que de forma, principalmente al transitar del Estado de Derecho al Estado Constitucional de Derecho con modelos democráticos que rigen sus instituciones, limitando el poder a través de una serie de contrapesos y teniendo un amplio catálogo de derechos humanos. En palabras de Luigi Ferrajoli los derechos constituyen la parte material de la democracia, ${ }^{22}$ y es en ese plano donde se hacen las siguientes reflexiones. Por ejemplo el neoconstitucionalismo ha traído consigo una forma particular de entender o dar una aproximación a los contenidos básicos de las constituciones, a la vez que se interpretan estos contenidos a la luz de una corriente iusnaturalista donde el derecho y la moral están vinculados y donde además los derechos también se conciben como valores; desde este tenor el neoconstitucionalismo aseguraría que los derechos fundamentales no pueden ser cualquier derecho y que tampoco es factible tener cualquier contenido en las constituciones. $^{23}$

La anterior premisa sirve de argumento para apuntalar las siguientes ideas: si se interpreta la Constitución a través de estos modelos de sistemas robustos de derechos, modelos democráticos nacidos principalmente a partir de la segunda posguerra, el sentido de lo constitucional debe tener otro enfoque, alejado de lo que Hans Kelsen hablaba sobre la Constitución formal y material, por lo tanto si no todo contenido debe estar figurado en la Constitución, el tema de las restricciones al ejercicio de los derechos humanos (aquellas que no estén de

\footnotetext{
${ }^{21}$ VILLASEÑOR GOYZUETA, Claudia Alejandra, Op. Cit., nota 8, p. 63.

${ }^{22}$ PAMPILlO BALIÑO, Juan Pablo, MUNIVE PÁEZ, Manuel Alexandro (coords.), Derecho Constitucional de los Derechos Humanos: Consideraciones entorno a la reforma del artículo $1^{\circ}$ Constitucional, México, Porrúa, Escuela Libre de Derecho, 2012, p. 636.

${ }^{23}$ SAlaZAR UGARTE, Pedro, Política y derecho. Derechos y garantías. Cinco ensayos latinoamericanos, $1^{\mathrm{a}}$ ed., Fontamara, México, 2013, p. 157.
} 
conformidad con la norma fundamental o bien trasgredan el núcleo esencial del derecho) encontraría un soporte filosófico mediante el cual sea factible considerar que una restricción podría ser inconstitucional/inconvencional a la vez, pues la perspectiva gira en torno a los derechos en el bloque constitucional/convencional, y no al formalismo bajo el que se concibe como ilimitada la actuación del poder reformador de la Constitución para plasmar cualquier contenido en la Constitución, volviéndolo así constitucional de manera automática.

Evidentemente este criterio no ha sido aceptado por la Suprema Corte aun cuando ya se han dado algunos desarrollos doctrinales sobre la constitución inconstitucional, enfocados algunos de ellos a las alteraciones al procedimiento de reforma o modificación del contenido, ${ }^{24}$ pero no así a la parte sustantiva de estos documentos supremos en los modelos democráticos. Para el Máximo Tribunal la Constitución no puede ser declarada a sí misma inconstitucional, criterio que ha prevalecido por más de dos décadas. ${ }^{25}$

Sin embargo no es posible interpretar de la misma manera la norma fundamental a raíz de la reforma del año 2011, pues como ha quedado señalado, el texto supremo se configuró como una Constitución de derechos, y no de cualquier tipo, sino de derechos humanos que además se integran desde otras fuentes al propio texto constitucional, por lo tanto el tema de las restricciones al ejercicio de los derechos humanos bajo la lógica de sus valores y contenido no debe quedar como una regla a priori para todos y cada uno de los límites, además se considera necesario dar pasos para reconocer que la Constitución en sus apartados puede ser inconstitucional por no estar de conformidad con ella misma en el entendido del bloque constitucional/convencional, y en ese sentido privilegiar los derechos y no sus restricciones.

\section{REFLEXIONES FINALES}

En este último apartado sólo se reafirmarán algunas de las consideraciones señaladas con anterioridad. En primer término se hace referencia a la necesidad de tomarse en serio la reforma en derechos humanos de 2011, y esto incluye no sólo a los operadores jurídicos en el sentido estricto de aquellos quienes realizan una labor en la impartición de justicia, sino a otros actores también, como los representantes del poder ejecutivo, los legisladores y desde luego a la ciudadanía, voces que necesariamente deben ser escuchadas si lo que se busca y desea es aterrizar en un sentido práctico los derechos humanos como reivindicaciones de todas las personas.

Referirnos a cristalizar la reforma implica una nueva interpretación del derecho, una necesaria y renovada lectura de las viejas estructuras que no permiten un verdadero acercamiento del contenido constitucional a la realidad social, cuyos integrantes exigen ver reflejadas sus libertades básicas en el día a día; por ello se destacó la idea del bloque constitucional

\footnotetext{
${ }^{24}$ Para un mejor desarrollo de este tema, sugiero consultar el artículo del Maestro Raúl González Schmal en http://biblio.juridicas.unam.mx/libros/1/130/18.pdf

${ }^{25}$ CONSTITUCION, TODAS SUS NORMAS TIENEN LA MISMA JERARQUIA Y NINGUNA DE ELLAS PUEDE DECLARARSE INCONSTITUCIONAL. De conformidad con el artículo 133 de la Constitución de la República todos sus preceptos son de igual jerarquía y ninguno de ellos prevalece sobre los demás, por lo que no puede aceptarse que algunas de sus normas no deban observarse por ser contrarias a lo dispuesto por otras. De ahí que ninguna de sus disposiciones pueda ser considerada inconstitucional. Por otro lado, la Constitución únicamente puede ser modificada o adicionada de acuerdo con los procedimientos que ella misma establece.
} 
de derechos al ser un punto de inflexión sobre el cual es posible dinamizar los derechos humanos para mejorar sus contenidos progresivamente y no regresivamente.

Con la interacción dentro del bloque constitucional de los derechos humanos no se debe perder de vista que aquél sirve de parámetro de validez y de interpretación de los mismos, pero a la vez impacta en el entendimiento de las obligaciones a cargo de las autoridades estatales, lo anterior merece un foco de atención importante porque desde las distintas sedes del poder podría violentarse el contenido de dichas obligaciones al diseñar restricciones que no cumplan con el contenido esencial de los derechos fundamentales o con el principio de proporcionalidad, o bien al interpretar de manera no restrictiva en el caso concreto de los límites se podría incurrir en una conducta que genere algún tipo de responsabilidad para el servidor público.

Por otro lado, de manera tajante se afirma que definir el significado de lo constitucional no debe sujetarse a un aspecto meramente formalista, sino que debe atender a la sustancia o esencia de los valores que se deciden privilegiar en un estado de derecho, y al respecto de ese tema se tendría que señalar ¿qué estado de derecho se busca? es decir uno que entiende la seguridad jurídica con interpretaciones donde se restrinja en mayor medida los derechos humanos y no así su progresivo mejoramiento y protección; o bien un estado de derecho que conciba la seguridad jurídica como lo señala José Luis Caballero Ochoa se consigue con un mayor y mejor ejercicio del sistema interpretativo de los derechos humano ${ }^{26}$ permitiendo que la igualdad y libertad jurídicas ocupen los primeros sitios en la agenda estatal.

En esa tesitura el sentido de lo que es o no constitucional con un enfoque material o de contenido apoyaría en algún momento determinado la posibilidad de superar una restricción a pesar de encontrarse de manera expresa en la Constitución.

Asimismo se debe recordar que la jurisprudencia derivada de la contradicción de tesis 293/2011 establece la necesaria prevalencia de la restricción expresa al ejercicio del derecho humano; sin embargo se cuestiona como se manifestó antes, de encontrarnos ante una regla a priori para ser utilizada en cada caso sin realizar ejercicios de ponderación y de proporcionalidad del límite al derecho, pues la repuesta será en todo momento la misma, predominará la restricción. En razón de ello es necesario ejecutar una modulación interpretativa teniendo como ejes la cláusula de interpretación conforme y el principio pro persona para restrictivamente interpretar el límite al derecho humano cuestionado, incluyendo desde luego la progresividad de los derechos para plantear la posibilidad de derrotar una restricción que no respete el núcleo esencial de los mismos. Lo cierto es que a través del control de convencionalidad se proyectará la manera en que los operadores jurídicos interactuarán con tales elementos al tener un caso concreto en el que resuelvan estos retos para la impartición de justicia.

Finalmente en adición a lo anterior, se deben idear otros mecanismos para analizar el tema de las restricciones constitucionales, sin iniciar necesariamente un litigio ante los tribunales con la finalidad de tener una garantía para el respeto de esa materia y sustancia en el contenido de las constituciones sobre todo cuando se habla de derechos, y evitar tener un poder reformador que diseñe un texto fundamental en los términos de Karl Loewenstein, es decir, una Constitución semántica alejada del modelo democrático ideal para un adecuado goce y disfrute de los derechos humanos.

\footnotetext{
${ }^{26}$ CABALlERO OCHOA, José Luis, Op. Cit., nota 3, nota preliminar, p. XXX.
} 
V. FUENTES DE INFORMACIÓN

CABALLERO OCHOA, José Luis, "La interpretación conforme, el modelo constitucional ante los tratados internacionales sobre derechos humanos y el control de convencionalidad" $2^{a}$ ed., Porrúa, México, 2014.

CARBONELL, Miguel, Elementos de derecho constitucional. $1^{\text {a }}$ ed., Fontamara, México, 2004 .

Teoría de la Constitución. Ensayos escogidos. $1^{\text {a }}$ ed., Porrúa, Universidad Nacional Autónoma de México, México, 2000.

, SALAZAR, Pedro (coords.). La reforma constitucional de derechos humanos. Un nuevo paradigma. $1^{\text {a }}$ ed., Porrúa, Universidad Nacional Autónoma de México, México, 2012.

Constitución Política de los Estados Unidos Mexicanos.

GÓNGORA MERA, Manuel Eduardo en http://www.nmrz.de/wpcontent/uploads/2009/11/ Bloque_Constitucionalidad_Argentina_impunidad.pdf. 15 de enero de 2015 .

GONZÁLEZ SCHMAL, Raul, en http://biblio.juridicas.unam.mx/libros/1/13o/18.pdf. 17 de enero de 2015.

MEDINA RODRÍGUEZ, Julio César, Derecho Constitucional Mexicano. $1^{\mathrm{a}}$ ed., Publicaciones administrativas Contables Jurídicas, México, 2012.

PACHECO PULIDO, Guillermo, La inmensidad del artículo $1^{\circ}$ de la Constitución Política de los Estados Unidos Meicanos, $1^{\text {a }}$ ed., Porrúa, México 2013.

PAMPILLO BALIÑO, Juan Pablo, MUNIVE PÁEZ, Manuel Alexandro (coords.), Derecho Constitucional de los Derechos Humanos: Consideraciones entorno a la reforma del artículo $1^{\circ}$ Constitucional, México, Porrúa, Escuela Libre de Derecho, 2012.

PELAYO MOLLER, Carlos, en http://www.miguelcarbonell.com/artman/uploads/1/las_reformas_en_derechos_humanos.pdf.17 de enero de 2015.

POST, Robert y REVA Siegel. Constitucionalismo democrático. Por una reconciliación entre Constitución y pueblo. $1^{\mathrm{a}}$ ed., Siglo Veintiuno Editores, Argentina, 2013.

RODRIGUEZ MANZO, Graciela en http://www2.scjn.gob.mx/red/coordinacion/Bloque\%2ode\%2oconstitucionalidad.pdf . 19 de enero de 2015.

SAlAZAR UGARTE, Pedro, Política y derecho. Derechos y garantías. Cinco ensayos latinoamericanos, $1^{\mathrm{a}}$ ed., Fontamara, México, 2013.

Semanario Judicial de la Federación.

SERRANO, Sandra y VAZQUEZ, Daniel, Los derechos humanos en acción, obligaciones y principios de derechos humanos. $1^{\mathrm{a}}$. ed. FLACSO, México, 2013.

UPRIMNY YEPES, Rodrigo en http://www.ejrlb.net/biblioteca2011/content/pdf/a16/1.pdf . 21 de enero de 2015. 
VILLASEÑOR GOYZUETA, Claudia Alejandra, Proporcionalidad y límites de los derechos fundamentales, teoría general y su reflejo en la jurisprudencia mexicana, $1^{\mathrm{a}}$ ed., Porrúa, Escuela Libre de Derecho y Universidad Complutense, México, 2011. 